\title{
Analysis of Reaction Times and Time-delays in an Intelligent HCI for a Smart Wheelchair
}

\author{
David Sanders \\ School of Mechanical and Design Engineering \\ University of Portsmouth, Portsmouth, UK \\ david.sanders@port.ac.uk \\ Giles tewkesbury \\ School of Mechanical and Design Engineering \\ University of Portsmouth, Portsmouth, UK \\ Giles.tewkesbury@port.ac.uk \\ Ian Rogers \\ Gems Sensors \\ Lennox Road, Basingstoke, RG22 4AW, UK \\ ian.rogers@gems-sensors.co.uk
}

\author{
Malik Haddad \\ School of Mechanical and Design Engineering \\ University of Portsmouth, Portsmouth, UK \\ malik.haddad@port.ac.uk \\ Nils Bausch \\ School of Energy and Electronic Engineering \\ University of Portsmouth, Portsmouth, UK \\ nils.bausch@port.ac.uk \\ Ya Huang \\ School of Mechanical and Design Engineering \\ University of Portsmouth, Portsmouth, UK \\ ya.huang@port.ac.uk
}

\begin{abstract}
The effect of introducing a delay between a joystick and a motor controller is investigated. Time-delays are introduced to a HCI for an intelligent wheelchair. The effects of the time-delays are then investigated. The ability of wheelchair users to complete tasks is considered. Two systems and two different ways for drivers to interact with their wheelchairs are considered in various situations. Wheelchair drivers were scrutinized while they completed a task with their wheelchair. Time-delay was introduced to investigate errors made by drivers undertaking tests with and without sensors and a computer system to assist them. As the delay was extended then more errors were made. When the time-delay was longer or when the wheelchair was moving through more complex situations then users did better when assisted by a sensor system. It is suggested that in simpler situations with a shorter time-delay then little sensor assistance was required but more assistance was needed in more complicated situations or when the time-delay was longer. So it might be better to vary the sensor support provided depending on the difficulties being encountered.
\end{abstract}

Keywords; Time-delay; intelligent; wheelchair; sensor; sonic

\section{INTRODUCTION}

Reaction times (RTs) are affected by: gender, physical fitness, tiredness, distraction, personality type, alcohol, age, and whether stimuli are visual or auditory. For example, patients with Parkinson's disease have longer RTs [1]. So, time-delays and their effect on performance of powered wheelchair users are investigated in this paper. Several different environments were investigated and users were provided with ultrasonic sensors that could assist them. Drivers used a joystick to control their chair and complete tasks with sensors assisting them and then with sensors disabled.
Some background is presented followed by a description of the system, then the tests are explained and some outcomes described. To close there is a discussion and some conclusions, including suggestions for work in the future.

A key conclusion was that wheelchair drivers may perform better in simple environments without any sensors assisting them [2-5]. Time-delays were introduced between the joystick and controller and with sensors disabled, user error rates increased appreciably as time-delays increased.

\section{BACKGROUND}

Slower reactions create time-delays. They can add distortions to control commands and control feedback [6] and can diminish performance [7]. Driving tends to compromise between stability margins and transparency [8]. A few control approaches for driving with unvarying delay times have been described [9] as well as some with varying time-delays [10].

$\mathrm{RT}$ is the time taken for messages to travel from sensors (for example eyes) to the brain and then on to a muscle (for example in the arm). Cells called neurons within the nervous system convey messages from and to the spinal cord and brain. Many factors affect RT. Patients with akinesia have significantly longer RTs $[11,12]$ and a lesion of the right basal ganglia causes a lengthening of RT [13]. RT has been studied widely [14] as it can be important, for example slower RT can cause driving accidents [14].

The flow of information in a vertebrate can be denoted as: Stimulus to Neuron to Spinal-Cord / Brain to Motor to Neuron to Response. A sensor neuron converts a stimulus into an electro-chemical signal within a sensory neuron that travels 
through the nervous system to a motor neuron. The motor neuron causes a muscle to contract or a gland to secrete.

Examples of things that affect RT are: Age, Right handed vs Left, Practice, Errors, Physical or Mental Fatigue, Distraction, Warning of Imminent Stimulation, Alcohol, Personality Type, Exercise, Threats, Stress, Stimulants, Learning Disorders, Brain Injury and Illness (for example minor upper respiratory tract infection).

This work was especially interested in the delay due to age, learning disorders, brain injury and illness, and less interested in personality type, gender, intelligence and alcohol. But the work is considering the effect of practice, making errors, physical or mental fatigue, stimulants and distraction on RTs and therefor delay.

Wheelchairs are often controlled with manual input transducers, for example joysticks [15] although other input transducers have been used, such as pointers [16,17], switches $[18,19]$, or a custom built Human Computer Interaction (HCI) in the form of a virtual reality interface [20]. The controllers usually interface between a lower current input device with a higher current actuator that typically drives a motor(s) connected to a wheel(s).

Disturbances can be introduced because of variances in the wheels or because of different reactions to a gradient or a surface $[18,19]$ or because of time-delays [9, 21-23] due to longer RTs [14]. Wheelchair drivers react to each disturbance and try to correct their steering.

\section{SYSTEM}

Ultrasonic transducers provide a reliable and economical solution for obstacle detection and ranging in close-proximity indoor environments [24]; they are robust and simple. $40 \mathrm{KHz}$ receiver / transmitter pairs were installed on the forward-face of the chair. The basic sound image provided a depiction of the surroundings.

A joystick and a parallel interface delivered signals to a digital controller (fitted with analogue interfaces). That drove current to the DC servo-amplifiers on a BobCat II wheelchair base. This allowed a powered wheelchair to be driven under the control of the computer that is by "fly-by-wire" [25]. The system sensed obstacles in the surroundings and modified control signals. The system is described in $[3,5,26,27]$. So the link between the chair and joystick was disengaged and a microcomputer inserted. The computer handled control data. The computer interrogated and activated the sensors and was programmed to adapt the path of the chair.

If required, data from the joystick could go directly to the powered wheelchair controller without modification. So the powered wheelchair reacted directly to input form joysticks. The software was assembled in the way presented in [28] with three main control levels (Servo, Strategic and Supervisory) as used in $[29,30]$.

Procedures employed these rules: Trajectories are only modified when necessary; Wheelchair movements must be controlled and smooth; User stays in overall control.

\section{TESTING}

Testing was undertaken to:

- Compare the system being jointly controlled using a mixture of computer and human control, with control by just a human user, with various time-delays applied between the joystick and controller to represent RTs.

- Record how long it took to achieve tasks with and without assistance from sensors as time-delays rose and gaps between obstacles were reduced in width.

- Record the minimum gap that a human wheelchair user could safely drive through as time-delays were increased, both with and without sensors.

Eight groups of tests were conducted for each course. Four without sensors or automatic assistance and four with sensors and with automatic assistance. An obstacle course was created for each test within various environments:

LABORATORY. Only two staggered objects placed on a flat floor with perpendicular walls around it.

SIMPLE CORRIDOR. Sloping and flat surfaces. Vertical walls. No doorways. Staggered objects placed on the floor.

COMPLICATED CORRIDOR. Sloping and flat surfaces. Vertical walls. Doorways. Some things on the walls (radiators, door surrounds etc). More obstacles in staggered formations.

OUTSIDE ENVIRONMENT. Complicated surroundings with sloping and flat surfaces. Sloping and vertical edges. Objects and obstacles positioned within outside environments.

Staff and students at the University of Portsmouth volunteered to drive during the testing. They were mainly students. A clear explanation of the study was provided (including benefits and risks) and the University Ethics Committee approved the testing procedure. There were 12 females and 38 males. The 50 contributors were $18-53$ years old (SD 5, Mean 23). Tests were repeated because the performance of human drivers was variable.

Drivers repeated test runs as many times as they wanted to, or available time allowed. So they were able to learn and perform at their best as the time-delay was extended. The experiments were viewed as enjoyable and subjects were competitive. People tried to beat others and their best times and performances. As time-delays became longer, numbers of failed test runs and numbers of successful test runs were logged. To be counted as a successful test run, a test course needed to be completed without any collisions. A failure was recorded if any collisions occurred.

A first set of experiments compared the ability of the human wheelchair drivers to drive around courses with obstacles set 90 $\mathrm{cm}$ apart; $10 \mathrm{~cm}$ broader than the chair $(5 \mathrm{~cm}$ each side). That was repeated with the intelligent computer systems assisting users. Then the two sets of tests were repeated with smaller and smaller gaps. When a test was successfully completed with a smaller gap then the user made at least one more attempt at the other test (with or without the computer and sensor system) to ensure the successful result was not just because the user had learned the way the system worked. If they again successfully 
completed the course passing through smaller gaps then at least one other attempt was made at the original course and with the original set up. Test runs started with a standing start at predetermined starting positions and gaps were checked by three researchers using measures. If not enough sets of results were logged (not enough result pairs) then those results were rejected.

Fig. 1 shows where delay was introduced before movement instructions were sent to the chair (h2). It was possible to delay the velocity command to the motors (v1) so that signal (vr) was delayed. $\mathrm{h}$ is total time-delay consisting of $\mathrm{h} 2$ (forward delay) and a backward delay h1. Fig.2 is a sketch of Complicated Corridor Number Three. Arrows show a general route for a wheelchair. Shaded blocks are the obstacles in the wheelchair path. Complicated Corridor Three also included two doubledoorways where one door was kept shut and the other open. That meant the chair had to be zig-zagged to pass through them.

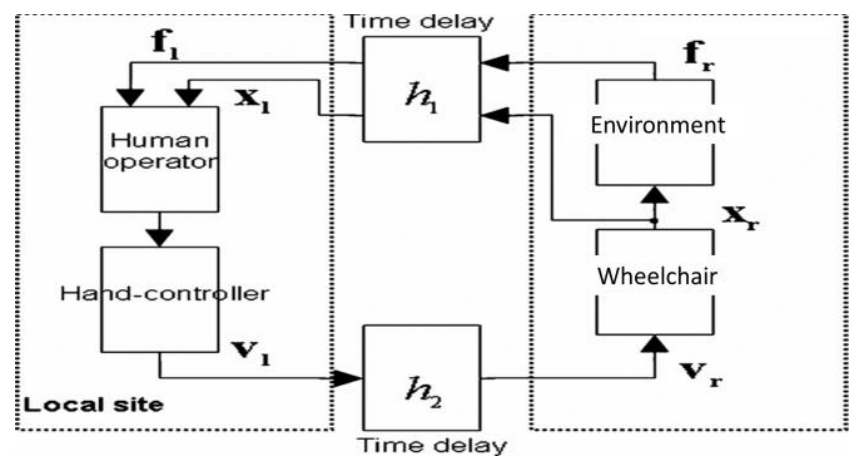

Figure 1. Delays in the system. Based on the system in [10]

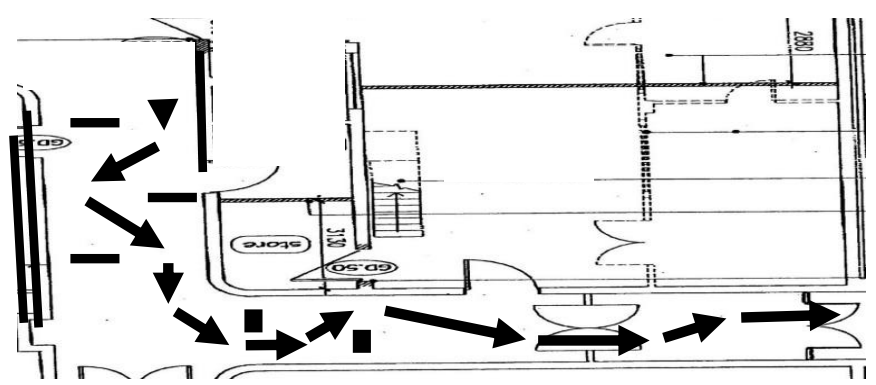

Figure 2. Complicated Corridor One

A camera was fixed on the chair to observe and record the tests while the driver used their joystick to guide their chair. Fig. 3 shows the scene from the camera as it moved though Complicated Corridor Two. At the end of the corridor, the researcher with the laboratory digital clock can be seen. Another researcher followed the chair with a stop watch. The series of pictures shows a successful test run with a delay of $2 \mathrm{~s}$.

\section{RESUlTS}

The wheelchair automatically avoided obstacles when the assistive computer systems were connected. There were some chaotic factors that affected the result, including variation in wheel position, slope or floor surface, or the trailing casters could send a chair off the desired path.

\section{A. Operation with and without sensors.}

Fig.4 displays the average of best time to finish a variety of routes. Average time to finish successful runs is shown on the vertical scale. Simple environments are to the left in each graph shown in the Figs, for example empty corridors and laboratory. The results show that drivers completed the simpler routes more quickly when they did not have any assistance from the sensors and computer system. More complex routes are shown to the right, for example outside routes and complicated corridors.
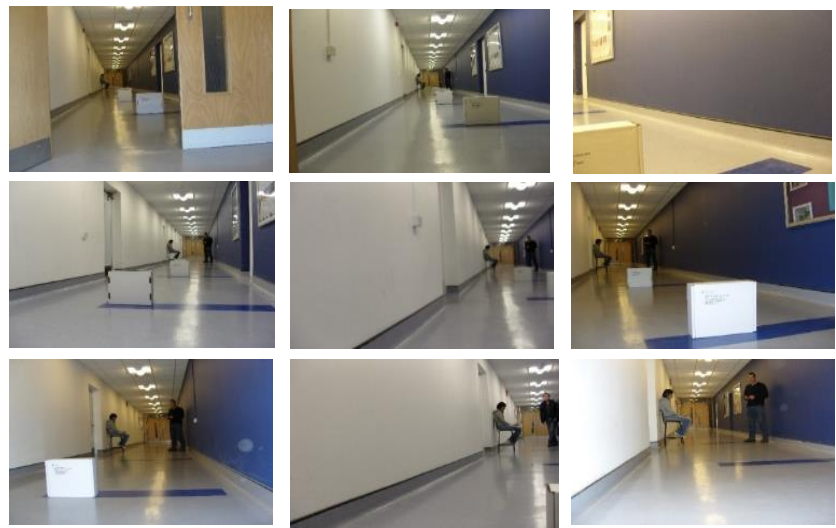

Figure 3. View from the camera on the wheelchair being assisted by the sensor system and moving though one of the complicated corridors

Wheelchair users finished the more complex routes faster when the sensor and computer system was connected and working. The lower graph shows the average of fastest times when a 1 s delay was introduced.
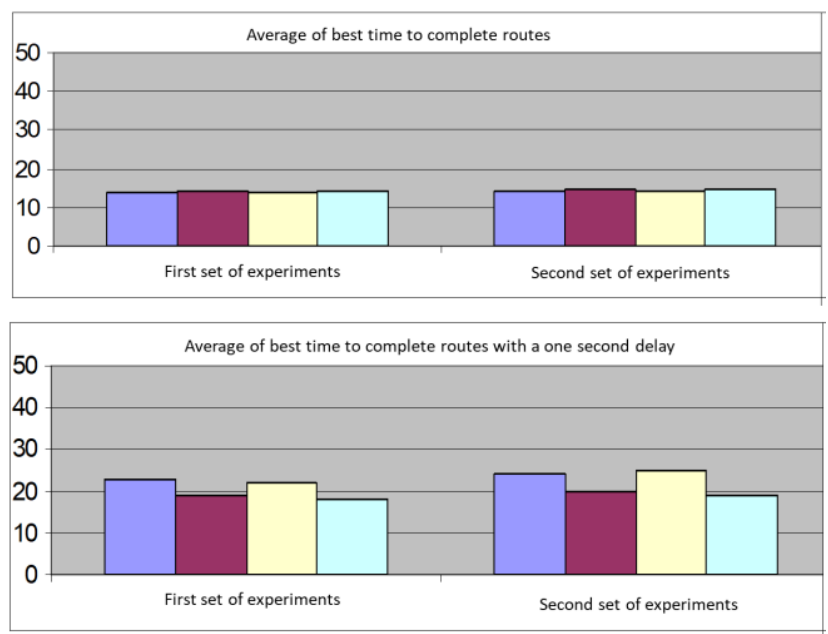

Figure 4. Average of best time to complete routes in Simple Corridor 2

Each time a test took place, gaps were reduced by $0.5 \mathrm{~cm}$. The thinnest set of gaps that a driver successfully navigated through were recorded with the number of failed and successful runs. Drivers completed courses with thinner gaps when utilizing the computer and sensors. Fig. 5 shows the average improvement in $\mathrm{cm}$ when using the sensors and microcomputer. The graph at the top is without any time-delay and the graph at the bottom is with a 1s delay. As simpler environments were changed into more complex environments or gaps changed to be thinner then drivers found it trickier to judge the width between obstacles. It 
was more difficult for them to pass through the thinner gaps. Drivers relied more on the sensor and computer systems. Drivers successfully drove through thinner gaps when the microcomputer and sensors were being used. Gradients, hills and surfaces had a tendency to turn the chairs and sensors became more useful in those cases. The microcomputer consistently corrected wheelchair angles and as time-delays increased then the results were more noticeable. Wheelchairs were driven faster through thinner gaps with the assistance from the sensors, especially when time-delays increased. Fig.5 shows the gaps acheived with and without the sensors engaged and without any delay (top). The bottom graph shows the gaps acheioved with and without the sensor systems engaged but with a delay of one second introduced.

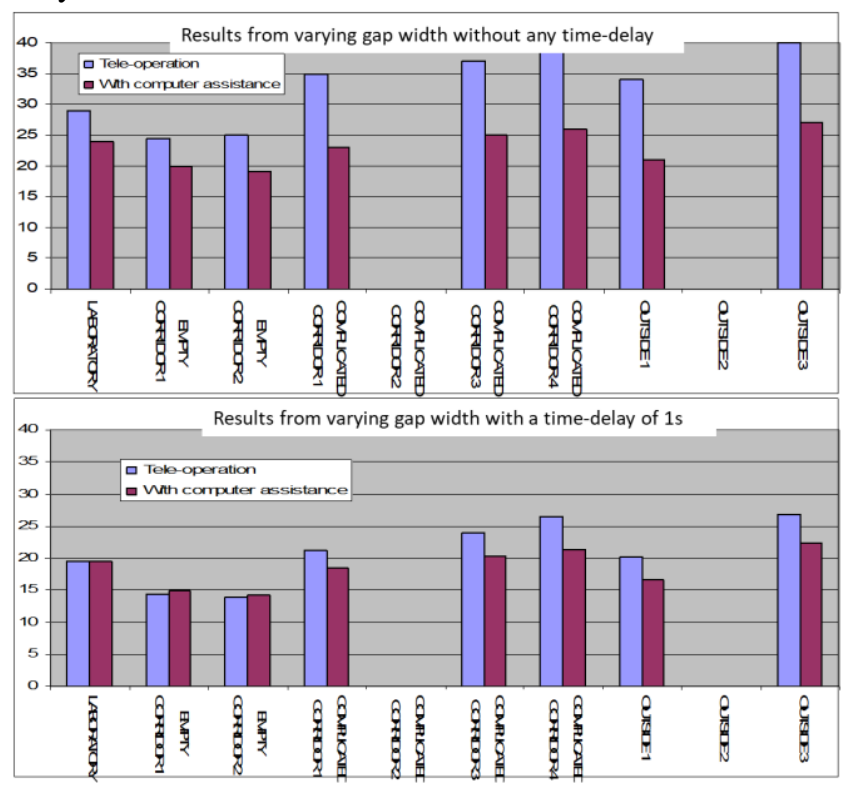

Figure 5. Results from varying gap width with a time-delay of 1s (bottom)

\section{B. Times to complete courses}

As gaps became thinner and time-delays increased, then the fastest time to complete routes and tasks was logged. When gaps widened, drivers completed courses faster without sensors and when gaps became thinner then wheelchair drivers completed routes and tasks faster with sensors and the microcomputer.

\section{Failure Rates}

Fig.6 displays failed and successful attempts with and without sensors and the microcomputer assisting drivers. The $\mathrm{x}$ axis is a list of different environments. Average numbers of failed and successful trials is to the left. The center bar shows percentage of failed attempts. The right shows the difference between failures when being assisted and when not. The top bar chart is when there was not any delay and at the bottom are the results with 1 s delays.

\section{DISCUSSION AND CONCLUSIONS}

In simpler situations, wheelchair drivers performed faster without sensors assisting them but in more complicated environments or with longer time delays then they were quicker with sensors assisting them. With wider gaps or in simpler situations then drivers consistently performed faster without help. As gaps reduced or environments became more complicated, or as time delays increased then drivers found driving more difficult and the sensors became more and more useful. When the situations became more complicated then drivers performed better with help from the sensors. As gaps reduced, assistive systems were consistently quicker than human drivers by themselves.

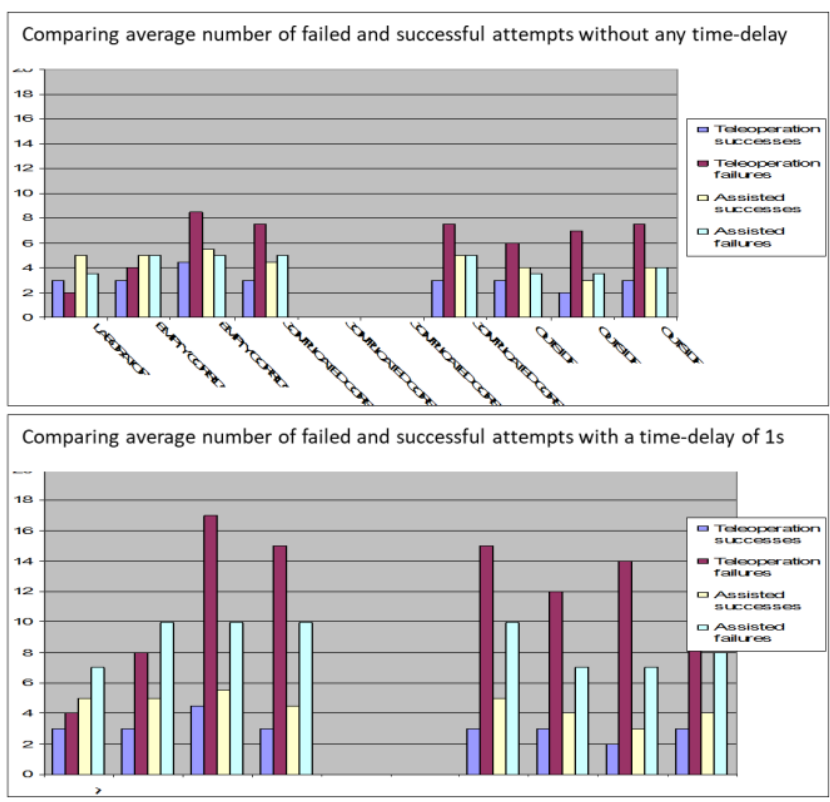

Figure 6. Comparing average number of failed and successful attempts with 1s delay

Time-delays were only introduced between the joystick and controller. Delays could be introduced elsewhere. Further statistical analysis could be conducted and delay compensation. A delay could have been introduced in two places but it was only introduced in displaying the camera view to the tele-operator. The system needs to be retested with a delay after the joystick and before transmitting the movement instructions to the mobile robot as results may then be significantly different. In any real system a delay would probably be present in both if it was present in one.

An implication of the results is that sensors should not be used in freely navigable regions with good views but should be reserved for more complicated situations.

Intelligence [31-34], input devices [35] and force sensing [36] could be included. Modelling [37-40] and decision making [40-45] are now being investigated for future application.

\section{ACKNOWLEDGMENT}

Research in this paper was funded by EPSRC grant EP/S005927/1 and supported by The Chailey Heritage Foundation and the University of Portsmouth.

\section{REFERENCES}

[1] I.T. Draper, R. Johns, "The disordered movement in parkinsonism and the effect of drug treatment”. Johns Hopkins Hosp 115, pp: 465-480, 1964. 
[2] D. Sanders, M. Langner, N. Bausch, Y. Huang, S.A. Khaustov, S. Simandjuntak, "Improving human-machine interaction for a powered wheelchair driver by using variable-switches and sensors that reduce wheelchair-veer". Intelligent Systems and Applications. Adv. Intell. Syst. Comput. vol. 1038, Springer, pp. 1173-1191 2019.

[3] D. Sanders, O.M. Okonor, M. Langner, M. Hassan Sayed, S.A. Khaustov, P.O. Omoarebun, "Using a simple expert system to assist a powered wheelchair user". Intelligent Systems and Applications. Adv. Intell. Syst. Comput. vol. 1037, Springer, pp. 662-379, 2019.

[4] D. Sanders, G. Tewkesbury, H. Parchizadeh, J.J. Robertson, P.O. Omoarebun, M. Malik, "Learning to drive with and without intelligent computer systems and sensors to assist". Adv. Intell. Syst. Comput. vol. 868, Springer, pp. 1171-1181, 2019.

[5] D. Sanders, A. Gegov, M. Haddad, F. Ikwan, D. Wiltshire, Y.C. Tan, “A rule-based expert system to decide on direction and speed of a powered wheelchair". Intelligent Systems and Applications: vol. 1. Adv. Intell. Syst. Comput. vol. 868, Springer, pp. 822-838, 2019

[6] P. Fiorini, R. Oboe, "Internet-based telerobotics: Problems and approaches", Proc' ICAR'97, Monterey, CA), pp. 765-770. Teleoperation of robots with delay 681. 1997.

[7] N Richard " Time delay systems: An overview of some recent advances and open problems", Automatica 39, pp 1667-1694. 2003.

[8] Lawrence DA. "Stability and transparency in bilateral teleoperation," IEEE Trans. Robot. Autom. 9 (5). 2003.

[9] W. Kim, B. Hannaford, A. Bejczy, "Force reflection and shared compliant control in operating telemanipulators with time delay", IEEE Trans. Robot. Autom. 8(2), 176-185. 1992.

[10] E. Slawinski, V. Mut, J.F. Postigo, "Teleoperation of robots with timevarying delay". Robotica (2006) volume 24, pp. 673-681. 2006.

[11] J. Brumlik, B. Boshes. "The mechanism of bradykinesia in parkinsonism”. Neurology (Minneap) 16:337-44. 1966.

[12] M. Joubert, A. Barbeau. "Akinesia in Parkinson's disease". Progress in Neurogenetics. Excerpta Medica Foundation pp:366-376. 1969

[13] M. Wiesendanger, P. Schneider, J.P. Villoz. "Electromyographic analysis of a rapid volitional movement". Am J Physical Med 48: 17-24. 1969.

[14] R.J. Kosinski, “A Literature Review on RT Kinds of RT Experiments." https://www.semanticscholar.org/paper/A-Literature-Review-onReaction-Time-Kinds-of-Time-Kosinski. 2012

[15] D. Sanders, A. Gegov, G. Tewkesbury, R. Khusainov, "Sharing driving between a vehicle driver and a sensor system using trust-factors to set control gains". Intelligent Systems and Applications: vol 1. Adv. Intell. Syst. Comput. vol. 868, Springer, pp. 1182-1195. 2019.

[16] D.A. Sanders "Comparing speed to complete progressively more difficult robot paths between human drivers and humans with sensor systems to assist". Assembly Automation. AA-08-057. 2009.

[17] D.A. Sanders, S. Urwin-Wright, G.E. Tewkesbury et al. Pointer device for thin-film transistor and cathode ray tube computer screens. Electronics Letters 41 (16), pp: 894-896. 2005

[18] I.J. Stott, D.A. Sanders "New powered wheelchair systems for the rehabilitation of some severely disabled users". Int Jnl of Rehabilitation Research 23 (3), pp: 149-153. 2000.

[19] D.A. Sanders "Controlling the direction of "walkie" type forklifts and pallet jacks on sloping ground”. Assemb Auto, 28 (4), pp 317-324. 2008.

[20] I.J. Stott, D.A. Sanders The use of virtual reality to train powered wheelchair users and test new wheelchair systems. Int Jnl of Rehabilitation Research 23 (4), pp 321-326. 2000.

[21] R.J. Anderson, M. Spong Bilateral control of teleoperators with time delay,” IEEE Trans. Autom. Control 34(5), pp: 494-501, 1989.

[22] J.Y. Chen, E.C. Haas, M.J. Barnes, "Human Performance Issues and User Interface Design for Teleoperated Robots". IEEE Trans on Systems, Man, and Cybernetics, Part C: Apps and Rev, 37, pp 1231-1245. 2007.

[23] D.A. Sanders, M. Langner, A.E. Gegov, D. Ndzi, H. Sanders, G.E. Tewkesbury, "Driver performance and their perception of system time lags when completing robot tasks". Proc 9th Int Conf Human Systems Interaction. IEEE, pp. 236-242. 2016.
[24] W. Gao, M Hinders "Robot sonar backscatter algorithm for automatically distinguishing walls, fences, and hedges". Int Jnl of Robotics Research 25 (2), pp 135-145. 2006.

[25] D.A. Sanders, A. Baldwin "X-by-wire technology". Total Vehicle Technology: Challenging current thinking, pp 3-12. 2001.

[26] D.A. Sanders "Comparing ability to complete simple nuclear rescue or maintenance tasks with a robot for a human driver and a human with a sensor system to assist". Advanced Robotics 8011. 2009.

[27] D. Sanders, "Analysis of failure rates with a robot between a human driver and a human with a sensor system to assist". Robotica. 2009.

[28] D. Sanders, "Microprocessing and microprogramming", 38, p 833. 1993.

[29] D.A. Sanders, "The modification of pre-planned manipulator paths to improve the gross motions associated with the pick and place task", Robotica, Vol. 13 pp.77-85. 1995.

[30] G.E. Tewlesbury, D.A. Sanders "A new robot command library which includes simulation”, Ind' Robot; an int' jnl, Vol 26, No 1, pp 39-48. 1999.

[31] D.A. Sanders, I.J. Stott IJ, "A new prototype intelligent mobility system to assist powered wheelchair users". Ind' Robot; an int' jnl, Vol 26, No 6, pp 466-475. 1999.

[32] M. Haddad, D. Sanders, N. Bausch, G. Tewkesbury, A. Gegov, M. Hassan Sayed, "Learning to make intelligent decisions using an Expert System for the intelligent selection of either PROMETHEE II or the Analytical Hierarchy Process". Intelligent Systems and Applications: vol 1. Adv. Intell. Syst. Comput. vol. 868, Springer, pp. 1303-1316 2019.

[33] D. Sanders, D.C. Robinson, M. Hassan Sayed, M.J.M. Haddad, A. Gegov, N. Ahmed, "Making decisions about saving energy in compressed ai systems using Ambient Intelligence and Artificial Intelligence". Adv. Intell. Syst. Comput. vol. 869, Springer, pp. 1229-1236, 2019.

[34] D. Sanders, "A pointer device for TFT display screens that determines position by detecting colours on the display using a colour sensor and an Artificial Neural Network". Displays. DISPLA-D-08-00006. 2009.

[35] D.A. Sanders S. Urwin-Wright, G.E. Tewkesbury et al. "Pointer device for thin-film transistor and cathode ray tube computer screens". Electronics Letters, 41 (16), pp 894-896. 2005.

[36] D. Sanders. Force sensing. Ind Rob -an int' jnl: 34 (4), pp 268-268. 2007.

[37] D.A. Sanders. "Recognising shipbuilding parts using ANNs and Fourier Descripors". IMechE Part B: Jnl of Eng Manf. JEM1382. 2009.

[38] D. Sanders, "Real time geometric modelling using models in an actuator space and Cartesian space", Jnl of Robotic Systems, vol. 12 No.1, pp.1928. 1995.

[39] D.A. Sanders. "Progress in Machine Intelligence". Industrial Robot - An International Journal Volume: 35 Issue: 6, Pages: 485-487. 2008.

[40] D. Sanders, Q. Wang, N. Bausch, Y. Huang, S.A. Khaustov, I. Popov, “A method to produce minimal real time geometric representations of moving obstacles. Intelligent Systems and Applications: vol 1. Adv. Intell. Syst. Comput. vol. 868, Springer, pp. 881-892, 2019.

[41] M.J.M Haddad, D. Sanders, A. Gegov, M. Hassan Sayed, Y. Huang, M Al-Mosawi, "Combining multiple criteria decision making with vector manipulation to decide on the direction for a powered wheelchair". Intelligent Systems and Applications. Adv. Intell. Syst. Comput. vol. 1037, Springer, pp. 680-693, 2019.

[42] M.J.M Haddad, D. Sanders, N. Bausch, "Selecting a robust decision making method to evaluate employee performance", International Journal of Management and Decision Making, vol. 18, no. 4, pp. 333-351. 2019.

[43] M.J.M Haddad, D. Sanders, G.E. Tewkesbury, "Selecting a discrete Multiple Criteria Decision Making method to decide on a corporate relocation", Archives of Business Research, vol. 7, no. 5, pp. 48-67. 2019.

[44] M.J.M. Haddad, D. Sanders, "Selecting a best compromise direction for a powered wheelchair using PROMETHEE', IEEE Trans on Neural Systems and Rehab Engineering, vol. 27, no. 2, pp. 228-235. 2019.

[45] M.J.M Haddad, D. Sanders, G. Tewkesbury, A. Gegov, M. Hassan Sayed, F.C. Ikwan, "Initial results from using Preference Ranking Organization METHods for Enrichment of Evaluations to help steer a powered wheelchair". Intelligent Systems and Applications. Adv. Intell. Syst. Comput. 1037, Springer, pp. 648-661, 2019 